\title{
On the Cauchy Problem for a Class of Weakly Dissipative One-Dimensional Shallow Water Equations
}

\author{
Jingjing $\mathrm{Xu}$ and Zaihong Jiang \\ Department of Mathematics, Zhejiang Normal University, Jinhua 321004, China \\ Correspondence should be addressed to Zaihong Jiang; jzhong790405@gmail.com
}

Received 21 June 2013; Accepted 22 August 2013

Academic Editor: Sining Zheng

Copyright (C) $2013 \mathrm{~J}$. Xu and Z. Jiang. This is an open access article distributed under the Creative Commons Attribution License, which permits unrestricted use, distribution, and reproduction in any medium, provided the original work is properly cited.

\begin{abstract}
We investigate a more general family of one-dimensional shallow water equations with a weakly dissipative term. First, we establish blow-up criteria for this family of equations. Then, global existence of the solution is also proved. Finally, we discuss the infinite propagation speed of this family of equations.
\end{abstract}

\section{Introduction}

Recently, in [1], the following one-dimensional shallow water equations were studied:

$$
y_{t}+a u_{x} y+b u y_{x}=0, \quad t>0, x \in \mathbb{R},
$$

where $u(x, t) \in \mathbb{R}$ and $y(x, t)=\left(1-\partial_{x}^{2}\right) u(x, t)$. A detailed description of the corresponding strong solution with the initial data $u_{0}$ was also given by them in [1].

When $a=\theta-1, b=\theta$, and $\lambda=0$, (1) reduces to $\theta$ equation which is studied by Ni and Zhou in [2].

When $a=2, b=1$, and $\lambda=0$, (1) reduces to the Camassa-Holm equation, which was derived physically by Camassa and Holm in [3] (found earlier by Fuchssteiner and Fokas [4] as a bi-Hamiltonian generalization of the KdV equation) by approximating directly the Hamiltonian for Euler's equations in the shallow water region with $u(x, t)$ representing the free surface above a flat bottom. The CamassaHolm equation is completely integrable and has infinite conservation laws. Local well-posedness for the initial datum $u_{0}(x) \in H^{s}$ with $s>3 / 2$ was proved in $[5,6]$. One of the remarkable features of Camassa-Holm equation is the presence of breaking waves and global solutions. Necessary and sufficient condition for wave breaking was established by Mckean [7] in 1998. A new and direct proof was also given in [8]. The solitary waves of Camassa-Holm equation are peaked solitons. The orbital stability of the peakons was shown by Constantin and Strauss in [9] (see also [10]). The property of propagation speed of solutions to the CamassaHolm equation, which was presented by Himonas and his collaborators in their work is worthy of being mentioned here [11].

The Degasperis-Procesi equation [12] and b-family equation [13] are the special cases with $a=3, b=1$, and $b=1$, respectively. There have been extensive studies on the two equations, (cf. $[14,15])$.

In this paper, we consider the following weakly dissipative one-dimensional shallow water equation:

$$
y_{t}+a u_{x} y+b u y_{x}+\lambda y=0, \quad t>0, x \in \mathbb{R},
$$

where $\lambda y=\lambda\left(u-u_{x x}\right)$ is the weakly dissipative term.

It is worth pointing out that many works have been done for related equations which have a weakly dissipative term (cf. [16-19]).

The paper is organized as follows. In Section 2, we establish the local well-posedness of the initial-value problem associated with (2) and present the precise blow-up scenario. Some blow-up results are given in Section 3. In Section 4, we establish a sufficient condition added on the initial data to guarantee global existence. We will consider the infinite propagation speed in Section 5. 


\section{Local Well-Posedness and Blow-Up Scenario}

In this section, we first establish the local well-posedness of (2) by using Kato's theory. Then, we provide the precise blowup scenario for solutions to (2).

System (2) is equivalent to the following system:

$$
u_{t}+b u u_{x}+\partial_{x} G *\left(\frac{a}{2} u^{2}+\frac{3 b-a}{2} u_{x}^{2}\right)+\lambda u=0,
$$

where $G(x)=(1 / 2) e^{-|x|}$, * means doing convolution.

Theorem 1. Given $u_{0} \in H^{s}(\mathbb{R}), s>3 / 2$, then there exist a $T$ and a unique solution $u$ to (2) such that

$$
u(x, t) \in C\left([0, T) ; H^{s}(\mathbb{R})\right) \cap C^{1}\left([0, T) ; H^{s-1}(\mathbb{R})\right) .
$$

To make the paper concise, we would like to omit the detailed proof, since one can find similar ones for these types of equations in [5].

\section{Blow-Up Phenomenon}

In this section, we will give some conditions to guarantee the finite time blowup. Motivated by Mckean's deep observation for the Camassa-Holm equation [7], we can consider the similar particle trajectory as

$$
\begin{gathered}
q_{t}=b u(q, t), \quad 0<t<T, x \in \mathbb{R}, \\
q(x, 0)=x, \quad x \in \mathbb{R},
\end{gathered}
$$

where $T$ is the lifespan of the solution: then $q$ is a diffeomorphism of the line. Taking derivative (5) with respect to $x$, we obtain

$$
\frac{d q_{t}}{d x}=q_{t x}=b u_{x}(q, t) q_{x}, \quad t \in(0, T)
$$

Therefore

$$
q_{x}(x, t)=\exp \left\{b \int_{0}^{t} u_{x}(q, s) d s\right\}, \quad q_{x}(x, 0)=1 .
$$

Hence, from (2), the following identity can be proved:

$$
y(q) q_{x}^{a / b}=y_{0}(x) e^{-\lambda t} .
$$

In fact, direct calculation yields

$$
\begin{aligned}
\frac{d}{d t}\left(y(q) q_{x}^{a / b}\right)=[ & y_{t}(q)+b u(q, t) y_{x}(q) \\
& \left.+a u_{x}(q, t) y(q)\right] q_{x}^{a / b}=-\lambda y q_{x}^{a / b} .
\end{aligned}
$$

Motivated by [19], we give the following theorem.

Theorem 2. Let $a-2 b>0, b>0$ : suppose that $u_{0} \in H^{2}(\mathbb{R})$, and there exists a $x_{0} \in \mathbb{R}$ such that $y_{0}\left(x_{0}\right)=\left(1-\partial_{x}^{2}\right) u_{0}\left(x_{0}\right)=0$,

$$
\begin{gathered}
y_{0} \geq 0(\neq \equiv 0), \quad \text { for } x \in\left(-\infty, x_{0}\right), \\
y_{0} \leq 0(\neq \equiv 0), \quad \text { for } x \in\left(x_{0}, \infty\right), \\
e^{-x_{0} \int_{-\infty}^{x_{0}} e^{\xi} y_{0}(\xi) d \xi>\frac{2 \lambda}{b},} \quad e^{x_{0}} \int_{x_{0}}^{\infty} e^{-\xi} y_{0}(\xi) d \xi<-\frac{2 \lambda}{b} .
\end{gathered}
$$

Then the corresponding solution $u(x, t)$ to (2) with $u_{0}$ as the initial datum blows up in finite time.

Proof. Suppose that the solution exists globally. From (8) and initial condition (10), we have $y\left(q\left(x_{0}, t\right), t\right)=0$ and

$$
\begin{aligned}
& y(q(x, t), t) \geq 0(\neq \equiv 0), \quad \text { for } x \in\left(-\infty, q\left(x_{0}, t\right)\right), \\
& y(q(x, t), t) \leq 0(\not \equiv 0), \quad \text { for } x \in\left(q\left(x_{0}, t\right), \infty\right),
\end{aligned}
$$

for all $t \geq 0$. Due to $u(x, t)=G * y(x, t)$, we can write $u(x, t)$ and $u_{x}(x, t)$ as

$$
\begin{gathered}
u(x, t)=\frac{1}{2} e^{-x} \int_{-\infty}^{x} e^{\xi} y(\xi, t) d \xi+\frac{1}{2} e^{x} \int_{x}^{\infty} e^{-\xi} y(\xi, t) d \xi \\
u_{x}(x, t)=-\frac{1}{2} e^{-x} \int_{-\infty}^{x} e^{\xi} y(\xi, t) d \xi+\frac{1}{2} e^{x} \int_{x}^{\infty} e^{-\xi} y(\xi, t) d \xi .
\end{gathered}
$$

Therefore,

$$
u_{x}^{2}(x, t)-u^{2}(x, t)=-\int_{-\infty}^{x} e^{\xi} y(\xi, t) d \xi \int_{x}^{\infty} e^{-\xi} y(\xi, t) d \xi
$$

for all $t>0$.

By direct calculation, for $x \leq q\left(x_{0}, t\right)$, we have

$$
\begin{aligned}
u_{x}^{2}(x, t)-u^{2}(x, t) & \\
= & -\int_{-\infty}^{x} e^{\xi} y(\xi, t) d \xi \int_{x}^{\infty} e^{-\xi} y(\xi, t) d \xi \\
= & -\left(\int_{-\infty}^{q\left(x_{0}, t\right)} e^{\xi} y(\xi, t) d \xi-\int_{x}^{q\left(x_{0}, t\right)} e^{\xi} y(\xi, t) d \xi\right) \\
& \times\left(\int_{q\left(x_{0}, t\right)}^{\infty} e^{-\xi} y(\xi, t) d \xi+\int_{x}^{q\left(x_{0}, t\right)} e^{-\xi} y(\xi, t) d \xi\right) \\
= & u_{x}^{2}\left(q\left(x_{0}, t\right), t\right)-u^{2}\left(q\left(x_{0}, t\right), t\right) \\
& -\int_{-\infty}^{x} e^{\xi} y(\xi, t) d \xi \int_{x}^{q\left(x_{0}, t\right)} e^{-\xi} y(\xi, t) d \xi \\
& +\int_{x}^{q\left(x_{0}, t\right)} e^{\xi} y(\xi, t) d \xi \int_{q\left(x_{0}, t\right)}^{\infty} e^{-\xi} y(\xi, t) d \xi \\
\leq & u_{x}^{2}\left(q\left(x_{0}, t\right), t\right)-u^{2}\left(q\left(x_{0}, t\right), t\right) .
\end{aligned}
$$

Similarly, for $x \geq q\left(x_{0}, t\right)$, we have

$$
u_{x}^{2}(x, t)-u^{2}(x, t) \leq u_{x}^{2}\left(q\left(x_{0}, t\right), t\right)-u^{2}\left(q\left(x_{0}, t\right), t\right) .
$$

So for any fixed $t$, combination of (15) and (16), we obtain

$$
u_{x}^{2}(x, t)-u^{2}(x, t) \leq u_{x}^{2}\left(q\left(x_{0}, t\right), t\right)-u^{2}\left(q\left(x_{0}, t\right), t\right),
$$

for all $x \in \mathbb{R}$. 
From the expression of $u_{x}(x, t)$ in terms of $y(x, t)$, differentiating $u_{x}\left(q\left(x_{0}, t\right), t\right)$ with respect to $t$, we have

$$
\begin{aligned}
\partial_{t} u_{x}\left(q\left(x_{0}, t\right), t\right) & \\
= & u_{x t}\left(q\left(x_{0}, t\right), t\right)+u_{x x}\left(q\left(x_{0}, t\right), t\right) q_{t}\left(q\left(x_{0}, t\right), t\right) \\
= & \frac{a}{2} u^{2}\left(q\left(x_{0}, t\right), t\right)+\frac{a-b}{2} u_{x}^{2}\left(q\left(x_{0}, t\right), t\right) \\
& -\lambda u_{x}\left(q\left(x_{0}, t\right), t\right)-G *\left(\frac{a}{2} u^{2}(x, t)+\frac{3 b-a}{2} u_{x}^{2}(x, t)\right) \\
= & G *\left(\frac{a}{2} u^{2}\left(q\left(x_{0}, t\right), t\right)+\frac{a-b}{2} u_{x}^{2}\left(q\left(x_{0}, t\right), t\right)\right. \\
& \left.\quad-\frac{a}{2} u^{2}(x, t)-\frac{3 b-a}{2} u_{x}^{2}(x, t)\right)-\lambda u_{x}\left(q\left(x_{0}, t\right), t\right) \\
= & G *\left(\frac { a - 2 b } { 2 } \left(u^{2}\left(q\left(x_{0}, t\right), t\right)-u_{x}^{2}\left(q\left(x_{0}, t\right), t\right)\right.\right. \\
& \left.\left.\quad-u^{2}(x, t)+u_{x}^{2}(x, t)\right)\right) \\
& +G *\left(b u^{2}\left(q\left(x_{0}, t\right), t\right)-\frac{b}{2} u_{x}^{2}\left(q\left(x_{0}, t\right), t\right)\right. \\
\leq & \left.\frac{b}{2} u^{2}\left(q\left(x_{0}^{2}, t\right), t\right)-\frac{b}{2} u_{x}^{2}\left(q\left(x_{0}, t\right), t\right)-\lambda u_{x}^{2}(x, t)\right)-\lambda u_{x}\left(q\left(x_{0}, t\right), t\right)
\end{aligned}
$$

where we have used (17), and the inequality $G *\left(u^{2}(x, t)+\right.$ $\left.(1 / 2) u_{x}^{2}(x, t)\right) \geq(1 / 2) u^{2}$. In addition, we also used the equation $u_{t x}+u u_{x x}-(a / 2) u^{2}-((b-a) / 2) u_{x}^{2}+G *\left((a / 2) u^{2}+\right.$ $\left.((3 b-a) / 2) u_{x}^{2}\right)+\lambda u_{x}=0$, which is obtained by differentiating equation (3).

For (11), we know that

$$
\begin{aligned}
& \left(u_{0 x}\left(x_{0}\right)+\frac{\lambda}{b}\right)^{2}-\left(u_{0}\left(x_{0}\right)+\frac{\lambda}{b}\right)^{2} \\
& =-e^{-x_{0}} \int_{-\infty}^{x_{0}} e^{\xi} y_{0}(\xi) d \xi \times\left(e^{x_{0}} \int_{x_{0}}^{\infty} e^{-\xi} y_{0}(\xi) d \xi+\frac{2 \lambda}{b}\right)>0, \\
& \left(u_{0 x}\left(x_{0}\right)+\frac{\lambda}{b}\right)^{2}-\left(u_{0}\left(x_{0}\right)-\frac{\lambda}{b}\right)^{2} \\
& =-e^{x_{0}} \int_{x_{0}}^{\infty} e^{-\xi} y_{0}(\xi) d \xi \times\left(e^{-x_{0}} \int_{-\infty}^{x_{0}} e^{\xi} y_{0}(\xi) d \xi-\frac{2 \lambda}{b}\right)>0 .
\end{aligned}
$$

Claim. $u_{x}\left(q\left(x_{0}, t\right), t\right)<0$ is decreasing. $\left(u\left(q\left(x_{0}, t\right), t\right)+\right.$ $\lambda / b)^{2}<\left(u_{x}\left(q\left(x_{0}, t\right), t\right)+\lambda / b\right)^{2}$ and $\left(u\left(q\left(x_{0}, t\right), t\right)-\lambda / b\right)^{2}<$ $\left(u_{x}\left(q\left(x_{0}, t\right), t\right)+\lambda / b\right)^{2}$, for all $t \geq 0$.

Suppose that there exists a $t_{0}$ such that $\left(u\left(q\left(x_{0}, t\right), t\right)+\right.$ $\lambda / b)^{2}<\left(u_{x}\left(q\left(x_{0}, t\right), t\right)+\lambda / b\right)^{2}$ and $\left(u\left(q\left(x_{0}, t\right), t\right)-\lambda / b\right)^{2}<$ $\left(u_{x}\left(q\left(x_{0}, t\right), t\right)+\lambda / b\right)^{2}$ on $\left[0, t_{0}\right)$; then $\left(u\left(q\left(x_{0}, t_{0}\right), t_{0}\right)+\right.$ $\lambda / b)^{2}=\left(u_{x}\left(q\left(x_{0}, t_{0}\right), t_{0}\right)+\lambda / b\right)^{2}$ or $\left(u\left(q\left(x_{0}, t_{0}\right), t_{0}\right)-\lambda / b\right)^{2}=$ $\left(u_{x}\left(q\left(x_{0}, t_{0}\right), t_{0}\right)+\lambda / b\right)^{2}$.

Now, let

$$
\begin{aligned}
I(t) & :=\frac{1}{2} e^{-q\left(x_{0}, t\right)} \int_{-\infty}^{q\left(x_{0}, t\right)} e^{\xi} y(\xi, t) d \xi \\
I I(t) & :=\frac{1}{2} e^{q\left(x_{0}, t\right)} \int_{q\left(x_{0}, t\right)}^{\infty} e^{-\xi} y(\xi, t) d \xi .
\end{aligned}
$$

Firstly, differentiating $I(t)$, we have

$$
\begin{aligned}
\frac{d I(t)}{d t}= & -\frac{b}{2} u\left(q\left(x_{0}, t\right), t\right) e^{-q\left(x_{0}, t\right)} \int_{-\infty}^{q\left(x_{0}, t\right)} e^{\xi} y(\xi, t) d \xi \\
& +\frac{1}{2} e^{-q\left(x_{0}, t\right)} \int_{-\infty}^{q\left(x_{0}, t\right)} e^{\xi} y_{t}(\xi, t) d \xi \\
= & \frac{b}{2} u\left(u_{x}-u\right)\left(q\left(x_{0}, t\right), t\right)-\frac{1}{2} e^{-q\left(x_{0}, t\right)} \\
& \times \int_{-\infty}^{q\left(x_{0}, t\right)} e^{\xi}\left(b u y_{x}+2 b u_{x} y\right. \\
& \left.\quad+\frac{a-2 b}{2}\left(u^{2}-u_{x}^{2}\right)_{x}+\lambda y\right) d \xi \\
\geq & \frac{b}{2} u\left(u_{x}-u\right)\left(q\left(x_{0}, t\right), t\right)+\frac{b}{4}\left(u^{2}+u_{x}^{2}-2 u u_{x}\right) \\
& \times\left(q\left(x_{0}, t\right), t\right)-\frac{\lambda}{2}\left(u-u_{x}\right)\left(q\left(x_{0}, t\right), t\right) \\
= & \frac{b}{4}\left(u_{x}^{2}-u^{2}\right)\left(q\left(x_{0}, t\right), t\right)-\frac{\lambda}{2}\left(u-u_{x}\right)\left(q\left(x_{0}, t\right), t\right) \\
= & \frac{b}{4}\left(u_{x}\left(q\left(x_{0}, t\right), t\right)+\frac{\lambda}{b}\right)^{2} \\
& -\frac{b}{4}\left(u\left(q\left(x_{0}, t\right), t\right)+\frac{\lambda}{b}\right)^{2}>0, \quad \text { on }\left[0, t_{0}\right) .
\end{aligned}
$$

Secondly, by the same argument, we obtain

$$
\begin{aligned}
\frac{d I I(t)}{d t}= & \frac{b}{2} u\left(q\left(x_{0}, t\right), t\right) e^{q\left(x_{0}, t\right)} \int_{q\left(x_{0}, t\right)}^{\infty} e^{-\xi} y(\xi, t) d \xi \\
& +\frac{1}{2} e^{q\left(x_{0}, t\right)} \int_{q\left(x_{0}, t\right)}^{\infty} e^{-\xi} y_{t}(\xi, t) d \xi \\
= & \frac{b}{2} u\left(u_{x}+u\right)\left(q\left(x_{0}, t\right), t\right)-\frac{1}{2} e^{q\left(x_{0}, t\right)} \\
& \times \int_{q\left(x_{0}, t\right)}^{\infty} e^{-\xi}\left(b u y_{x}+2 b u_{x} y\right. \\
& \left.+\frac{a-2 b}{2}\left(u^{2}-u_{x}^{2}\right)_{x}+\lambda y\right) d \xi
\end{aligned}
$$




$$
\begin{aligned}
\leq & \frac{b}{2} u\left(u_{x}+u\right)\left(q\left(x_{0}, t\right), t\right) \\
& -\frac{b}{4}\left(u^{2}+u_{x}^{2}+2 u u_{x}\right)\left(q\left(x_{0}, t\right), t\right) \\
& -\frac{\lambda}{2}\left(u_{x}+u\right)\left(q\left(x_{0}, t\right), t\right) \\
= & -\frac{b}{4}\left(u_{x}^{2}-u^{2}\right)\left(q\left(x_{0}, t\right), t\right) \\
& -\frac{\lambda}{2}\left(u_{x}+u\right)\left(q\left(x_{0}, t\right), t\right) \\
= & -\frac{b}{4}\left(u_{x}\left(q\left(x_{0}, t\right), t\right)+\frac{\lambda}{b}\right)^{2} \\
& +\frac{b}{4}\left(u\left(q\left(x_{0}, t\right), t\right)-\frac{\lambda}{b}\right)^{2}<0, \quad \text { on }\left[0, t_{0}\right) .
\end{aligned}
$$

Therefore, it follows from (21), (22), and the continuity property of ODEs that

$$
\begin{aligned}
& \left(u_{x}\left(q\left(x_{0}, t\right), t\right)+\frac{\lambda}{b}\right)^{2}-\left(u\left(q\left(x_{0}, t\right), t\right)+\frac{\lambda}{b}\right)^{2} \\
& =-4 I(t)\left(I I(t)+\frac{\lambda}{b}\right)>-4 I(0)\left(I I(0)+\frac{\lambda}{b}\right)>0 \\
& \left(u_{x}\left(q\left(x_{0}, t\right), t\right)+\frac{\lambda}{b}\right)^{2}-\left(u\left(q\left(x_{0}, t\right), t\right)-\frac{\lambda}{b}\right)^{2} \\
& =-4\left(I(t)-\frac{\lambda}{b}\right) I I(t)>-4\left(I(0)-\frac{\lambda}{b}\right) I I(0)>0
\end{aligned}
$$

for all $t>0$. This implies that $t_{0}$ can be extended to the infinity.

Moreover, using (21) and (22) again, we have the following equation for $\left[2\left(u_{x}+\lambda / b\right)^{2}-(u+\lambda / b)^{2}-(u-\lambda / b)^{2}\right]\left(q\left(x_{0}, t\right), t\right)$ :

$$
\begin{aligned}
& \frac{d}{d t}\left[2\left(u_{x}+\frac{\lambda}{b}\right)^{2}-\left(u+\frac{\lambda}{b}\right)^{2}-\left(u-\frac{\lambda}{b}\right)^{2}\right]\left(q\left(x_{0}, t\right), t\right) \\
& =-4 \frac{d}{d t}\left[I(t)\left(I I(t)+\frac{\lambda}{b}\right)\right]-4 \frac{d}{d t}\left[\left(I(t)-\frac{\lambda}{b}\right) I I(t)\right] \\
& \geq-b\left[\left(u_{x}+\frac{\lambda}{b}\right)^{2}-\left(u+\frac{\lambda}{b}\right)^{2}\right]\left(q\left(x_{0}, t\right), t\right)\left(I I(t)+\frac{\lambda}{b}\right) \\
& +b\left[\left(u_{x}+\frac{\lambda}{b}\right)^{2}-\left(u-\frac{\lambda}{b}\right)^{2}\right]\left(q\left(x_{0}, t\right), t\right) I(t) \\
& -b\left[\left(u_{x}+\frac{\lambda}{b}\right)^{2}-\left(u+\frac{\lambda}{b}\right)^{2}\right]\left(q\left(x_{0}, t\right), t\right) I I(t) \\
& +b\left[\left(u_{x}+\frac{\lambda}{b}\right)^{2}-\left(u-\frac{\lambda}{b}\right)^{2}\right]\left(q\left(x_{0}, t\right), t\right)\left(I(t)-\frac{\lambda}{b}\right)
\end{aligned}
$$

$$
\begin{aligned}
=b( & -\frac{\lambda}{b}\left[2\left(u_{x}+\frac{\lambda}{b}\right)^{2}-\left(u+\frac{\lambda}{b}\right)^{2}-\left(u-\frac{\lambda}{b}\right)^{2}\right] \\
& \times\left(q\left(x_{0}, t\right), t\right)-u_{x}\left(q\left(x_{0}, t\right), t\right) \\
& \times\left(2\left(u_{x}\left(q\left(x_{0}, t\right), t\right)+\frac{\lambda}{b}\right)^{2}\right) \\
& \left.+2\left(u+\frac{\lambda}{b}\right)^{2} I I(t)-2\left(u-\frac{\lambda}{b}\right)^{2} I(t)\right) \\
=b( & \left(u_{x}\left(q\left(x_{0}, t\right), t\right)+\frac{\lambda}{b}\right) \\
& \times\left[2\left(u_{x}+\frac{\lambda}{b}\right)^{2}-\left(u+\frac{\lambda}{b}\right)^{2}-\left(u-\frac{\lambda}{b}\right)^{2}\right] \\
\times & \times\left(q\left(x_{0}, t\right), t\right)-u_{x}\left(q\left(x_{0}, t\right), t\right) \\
\times-b\left(u_{x}\left(q\left(x_{0}, t\right), t\right)+\frac{\lambda}{b}\right) & {\left.\left[\left(u_{x}+\frac{\lambda}{b}\right)^{2}-\left(u+\frac{\lambda}{b}\right)^{2}-\left(u-\frac{\lambda}{b}\right)^{2}\right]\left(q\left(x_{0}, t\right), t\right),\left(u-\frac{\lambda}{b}\right)^{2}\right]\left(q\left(x_{0}, t\right), t\right) } \\
& \left.\times 2\left(u+\frac{\lambda}{b}\right)^{2} I I(t)-2\left(u-\frac{\lambda}{b}\right)^{2} I(t)\right) \\
& {[(u)}
\end{aligned}
$$

where we use $u_{x}\left(q\left(x_{0}, t\right), t\right)=-I(t)+I I(t)$.

Now, recalling (18), we have

$$
\begin{aligned}
\partial_{t} u_{x} & \left(q\left(x_{0}, t\right), t\right)\left(q\left(x_{0}, t\right), t\right) \\
\leq & \frac{b}{2} u^{2}\left(q\left(x_{0}, t\right), t\right)-\frac{b}{2} u_{x}^{2}\left(q\left(x_{0}, t\right), t\right)-\lambda u_{x} \\
= & \frac{b}{4}\left[\left(u+\frac{\lambda}{b}\right)^{2}+\left(u-\frac{\lambda}{b}\right)^{2}-2\left(u_{x}+\frac{\lambda}{b}\right)^{2}\right] \\
& \times\left(q\left(x_{0}, t\right), t\right) .
\end{aligned}
$$

Putting (25) into (24), it yields

$$
\begin{gathered}
\frac{d}{d t}\left[2\left(u_{x}+\frac{\lambda}{b}\right)^{2}-\left(u+\frac{\lambda}{b}\right)^{2}-\left(u-\frac{\lambda}{b}\right)^{2}\right]\left(q\left(x_{0}, t\right), t\right) \\
\geq \frac{b^{2}}{4}\left[2\left(u_{x}+\frac{\lambda}{b}\right)^{2}-\left(u+\frac{\lambda}{b}\right)^{2}-\left(u-\frac{\lambda}{b}\right)^{2}\right] \\
\times\left(q\left(x_{0}, t\right), t\right) \\
\times\left(\int_{0}^{t}\left[2\left(u_{x}+\frac{\lambda}{b}\right)^{2}-\left(u+\frac{\lambda}{b}\right)^{2}-\left(u-\frac{\lambda}{b}\right)^{2}\right]\right. \\
\left.\times\left(q\left(x_{0}, \tau\right), \tau\right) d \tau-4 u_{0 x}\left(x_{0}\right)-\frac{4 \lambda}{b}\right) .
\end{gathered}
$$


Before finishing the proof, we need the following technical lemma.

Lemma 3 (see [15]). Suppose that $\Psi(t)$ is twice continuously differential satisfying

$$
\begin{gathered}
\Psi^{\prime \prime}(t) \geq C_{0} \Psi^{\prime}(t) \Psi(t), \quad t>0, C_{0}>0, \\
\Psi(t)>0, \quad \Psi^{\prime}(t)>0 .
\end{gathered}
$$

Then $\psi(t)$ blows up in finite time. Moreover the blow-up time can be estimated in terms of the initial datum as

$$
T \leq \max \left\{\frac{2}{C_{0} \Psi(0)}, \frac{\Psi(0)}{\Psi^{\prime}(0)}\right\} .
$$

Let $\Psi(t)=\int_{0}^{t}\left[2\left(u_{x}+\lambda / b\right)^{2}-(u+\lambda / b)^{2}-(u-\right.$ $\left.\lambda / b)^{2}\right]\left(q\left(x_{0}, \tau\right), \tau\right) d \tau-4 u_{0 x}\left(x_{0}\right)-4 \lambda / b$; then (26) is an equation of type (27) with $C_{0}=b^{2} / 4$. The proof is complete by applying Lemma 3.

Remark 4 . When $b=1$, Theorem 2 reduces to the result in [19].

Theorem 5. Let $a=2 b>0$. Suppose that $u_{0} \in H^{2}(\mathbb{R})$ and there exists a $x_{0} \in \mathbb{R}$ such that $y_{0}\left(x_{0}\right)=\left(1-\partial_{x}^{2}\right) u_{0}\left(x_{0}\right)=0$,

$$
e^{-x_{0}} \int_{-\infty}^{x_{0}} e^{\xi} y_{0}(\xi) d \xi>\frac{2 \lambda}{b}, \quad e^{x_{0}} \int_{x_{0}}^{\infty} e^{-\xi} y_{0}(\xi) d \xi<-\frac{2 \lambda}{b}
$$

Then the corresponding solution $u(x, t)$ to (2) with $u_{0}$ as the initial datum blows up in finite time.

Proof. We easily obtain

$$
u_{t x}+b u u_{x x}-\frac{a}{2} u^{2}+\frac{b}{2} u_{x}^{2}+G *\left(\frac{a}{2} u^{2}+\frac{b}{2} u_{x}^{2}\right)+\lambda u_{x}=0 .
$$

Differentiating $u_{x}$ at the point $\left(q\left(x_{0}, t\right), t\right)$ with respect to $t$, we get

$$
\begin{aligned}
& \frac{d}{d t} u_{x}\left(q\left(x_{0}, t\right), t\right) \\
& \quad \leq \frac{b}{2} u^{2}\left(q\left(x_{0}, t\right), t\right)-\frac{b}{2} u_{x}^{2}\left(q\left(x_{0}, t\right), t\right)-\lambda u_{x}\left(q\left(x_{0}, t\right), t\right) .
\end{aligned}
$$

Process of the proof is similar to Theorem 2. Thus to be concise, we omit the detailed proof.

When $a=2 b>0, \lambda=0$, using $\widetilde{u}(x, t)=b u(x, t),(2)$ can be reformulated into

$$
\tilde{u}_{x}+\tilde{u}_{x x t}+3 \tilde{u} \tilde{u}_{x}-2 \tilde{u}_{x} \tilde{u}_{x x}-\tilde{u} \tilde{u}_{x x x}=0,
$$

which is the well-known Camassa-Holm equation. Meanwhile, we also find that the condition in Theorem 5 can be reformulated into

$$
\int_{-\infty}^{x_{0}} e^{\xi} \tilde{y}_{0}(\xi) d \xi>0, \quad \int_{x_{0}}^{\infty} e^{-\xi} \tilde{y}_{0}(\xi) d \xi<0,
$$

which is one of the sufficient conditions to guarantee blow-up add-on initial data for the Camassa-Holm equation.

So, we show the necessary and sufficient condition for the special case $a=2 b>0$ and $\lambda=0$ in the following theorem.

Theorem 6. When $a=2 b>0$ and $\lambda=0$, then the nonlinear wave equation (2) breaks if and only if some portion of the positive part of $y_{0}(x)$ lies to the left of some portion of its negative part.

Proof. As studied in [1], when $a=2 b>0$ and $\lambda=0$, rewriting (2) yields

$$
u_{x}+u_{x x t}+3 b u u_{x}-2 b u_{x} u_{x x}-b u u_{x x x}=0 .
$$

Recalling Mckean's theorem in [7], (32) breaks if and only if some portion of the positive part of $\tilde{y}_{0}(x, t)=\left(1-\partial_{x}^{2}\right) \tilde{u}_{0}$ lies to the left of some portion of its negative part.

So (34) breaks if and only if some portion of the positive part of $b y_{0}(x, t)=b\left(1-\partial_{x}^{2}\right) u_{0}$ lies to the left of some portion of its negative part.

This completes the proof.

Remark 7. Mckean's theorem [7] is for the special case $a=2$, $b=1$. Condition $a=2 b$ here is more general. However, the necessary and sufficient condition for (2) is still a challenging problem for us at present.

\section{Global Existence}

Now, let us try to find a condition for global existence. Unfortunately, When $a \neq 2 b$, like the Degasperis-Procesi equation [12], only the following easy one can be proved at present.

Theorem 8. Suppose that $u_{0} \in H^{3}(\mathbb{R})$, and $y_{0}=\left(1-\partial_{x}^{2}\right) u_{0}$ is one sign. Then the corresponding solution to (2) exists globally.

Proof. We can assume that $y_{0} \geq 0$. It is sufficient to prove that $u_{x}(x, t)$ has a lower and upper bound for all $t$. In fact,

$$
u_{x}(x, t)=-\frac{1}{2} e^{-x} \int_{-\infty}^{x} e^{\xi} y(\xi, t) d \xi+\frac{1}{2} e^{x} \int_{x}^{\infty} e^{-\xi} y(\xi, t) d \xi .
$$

Therefore, we have

$$
\begin{aligned}
u_{x}(x, t) & \geq-\frac{1}{2} e^{-x} \int_{-\infty}^{x} e^{\xi} y(\xi, t) d \xi \geq-\frac{1}{2} \int_{-\infty}^{x} y(\xi, t) d \xi \\
& \geq-\frac{1}{2} \int_{-\infty}^{\infty} y(\xi, t) d \xi=-\frac{1}{2} \int_{-\infty}^{\infty} y_{0}(\xi, t) d \xi, \\
u_{x}(x, t) & \leq \frac{1}{2} e^{x} \int_{x}^{\infty} e^{-\xi} y(\xi, t) d \xi \leq \frac{1}{2} \int_{x}^{\infty} y(\xi, t) d \xi \\
& \leq \frac{1}{2} \int_{-\infty}^{\infty} y(\xi, t) d \xi=\frac{1}{2} \int_{-\infty}^{\infty} y_{0}(\xi, t) d \xi .
\end{aligned}
$$

This completes the proof. 


\section{Infinite Propagation Speed}

In this section, we will give a more detailed description on the corresponding strong solution $u(x, t)$ to (2) in its life span with initial data $u_{0}(x)$ being compactly supported. The main theorem reads as follows.

Theorem 9. Let $0<a \leq 3 b$. Assume that for some $T \geq 0$ and $s \geq 5 / 2, u \in C\left([0, T) ; H^{s}(\mathbb{R})\right)$ is a strong solution of $(2)$. If $u_{0}(x)=u(x, 0)$ has compact support $[a, c]$, then for $t \in(0, T)$, one has

$$
u(x, t)= \begin{cases}L(t) e^{-x}, & \text { for } x>q(c, t), \\ l(t) e^{-x}, & \text { for } x<q(a, t),\end{cases}
$$

where $L(t)$ and $l(t)$ denote continuous nonvanishing functions, with $L(t)>0$ and $l(t)<0$ for $t \in(0, T)$. Furthermore, $L(t)$ is strictly increasing function, while $l(t)$ is strictly decreasing function.

Proof. Since $u_{0}$ has compact support in $x$ in $[a, c]$, from (8), so does $y(, t)$ has compact support in $x$ in $[q(a, t), q(c, t)]$ in its lifespan. Hence the following functions are well-defined:

$$
E(t)=\int_{\mathbb{R}} e^{x} y(x, t) d x, \quad F(t)=\int_{\mathbb{R}} e^{-x} y(x, t) d x,
$$

with

$$
E_{0}=\int_{\mathbb{R}} e^{x} y_{0}(x) d x=0, \quad F_{0}=\int_{\mathbb{R}} e^{-x} y_{0}(x) d x=0 .
$$

Thus, for $x>q(c, t)$, we obtain

$$
\begin{aligned}
u(x, t) & =\frac{1}{2} e^{-|x|} * y(x, t) \\
& =\frac{1}{2} e^{-x} \int_{q(a, t)}^{q(b, t)} e^{\tau} y(\tau, t) d \tau=\frac{1}{2} e^{-x} E(t) .
\end{aligned}
$$

Similarly, for $x<q(a, t)$, we have

$$
\begin{aligned}
u(x, t) & =\frac{1}{2} e^{-|x|} * y(x, t)=\frac{1}{2} e^{x} \int_{q(a, t)}^{q(b, t)} e^{-\tau} y(\tau, t) d \tau \\
& =\frac{1}{2} e^{x} F(t) .
\end{aligned}
$$

Hence, as consequences of (40) and (41), we get

$$
\begin{array}{r}
u(x, t)=-u_{x}(x, t)=u_{x x}(x, t)=\frac{1}{2} e^{-x} E(t), \\
\text { as } x>q(c, t), \\
u(x, t)=u_{x}(x, t)=u_{x x}(x, t)=\frac{1}{2} e^{x} F(t), \\
\text { as } x<q(a, t) .
\end{array}
$$

On the other hand,

$$
\frac{d E(t)}{d t}=\int_{\mathbb{R}} e^{x} y_{t}(x, t) d x
$$

It is easy to get

$$
\begin{aligned}
y_{t}= & -b u u_{x}+b\left(u u_{x}\right)_{x x}-\partial_{x}\left(\frac{a}{2} u^{2}+\frac{3 b-a}{2} u_{x}^{2}\right) \\
& -\lambda u+\lambda u_{x x} .
\end{aligned}
$$

Putting the identity (44) into $d E(t) / d t$, we have

$$
\begin{aligned}
& \frac{d E(t)}{d t} \\
& =\int_{\mathbb{R}} e^{x}\left(-b u u_{x}+b\left(u u_{x}\right)_{x x}-\partial_{x}\left(\frac{a}{2} u^{2}+\frac{3 b-a}{2} u_{x}^{2}\right)\right) d x \\
& \quad+\int_{\mathbb{R}} e^{x}\left(-\lambda u+\lambda u_{x x}\right) d x \\
& =\int_{\mathbb{R}} e^{x}\left(\frac{a}{2} u^{2}+\frac{3 b-a}{2} u_{x}^{2}\right) d x
\end{aligned}
$$

where we have used (42).

Therefore, in the lifespan of the solution, we get

$$
E(t)=\int_{0}^{t} \int_{\mathbb{R}} e^{x}\left(\frac{a}{2} u^{2}+\frac{3 b-a}{2} u_{x}^{2}\right)(x, \tau) d x d \tau>0 .
$$

By the same argument, one can check that the following identity for $F(t)$ is true:

$$
F(t)=-\int_{0}^{t} \int_{\mathbb{R}} e^{-x}\left(\frac{a}{2} u^{2}+\frac{3 b-a}{2} u_{x}^{2}\right)(x, \tau) d x d \tau<0 .
$$

In order to complete the proof, it is sufficient to let $L(t)=$ $(1 / 2) E(t)$ and $l(t)=(1 / 2) F(t)$, respectively.

\section{Acknowledgments}

This work is partially supported by Zhejiang Innovation Project (T200905), ZJNSF (Grant no. R6090109), and NSFC (Grant no. 10971197 and 11101376).

\section{References}

[1] Z. Jiang and S. Hakkaev, "Wave breaking and propagation speed for a class of one-dimensional shallow water equations," Abstract and Applied Analysis, vol. 2011, Article ID 647368, 15 pages, 2011.

[2] L. Ni and Y. Zhou, "Wave breaking and propagation speed for a class of nonlocal dispersive $\theta$-equations," Nonlinear Analysis: Real World Applications, vol. 12, no. 1, pp. 592-600, 2011.

[3] R. Camassa and D. D. Holm, "An integrable shallow water equation with peaked solitons," Physical Review Letters, vol. 71, no. 11, pp. 1661-1664, 1993.

[4] B. Fuchssteiner and A. S. Fokas, "Symplectic structures, their Bäcklund transformations and hereditary symmetries," Physica D, vol. 4, no. 1, pp. 47-66, 1981-1982.

[5] A. Constantin and J. Escher, "Well-posedness, global existence, and blowup phenomena for a periodic quasi-linear hyperbolic equation," Communications on Pure and Applied Mathematics, vol. 51, no. 5, pp. 475-504, 1998. 
[6] Y. A. Li and P. J. Olver, "Well-posedness and blow-up solutions for an integrable nonlinearly dispersive model wave equation," Journal of Differential Equations, vol. 162, no. 1, pp. 27-63, 2000.

[7] H. P. McKean, "Breakdown of a shallow water equation," The Asian Journal of Mathematics, vol. 2, no. 4, pp. 867-874, 1998.

[8] Z. Jiang, L. Ni, and Y. Zhou, "Wave breaking of the CamassaHolm equation," Journal of Nonlinear Science, vol. 22, no. 2, pp. 235-245, 2012.

[9] A. Constantin and W. A. Strauss, "Stability of peakons," Communications on Pure and Applied Mathematics, vol. 53, no. 5, pp. 603-610, 2000.

[10] Y. Zhou, "Stability of solitary waves for a rod equation," Chaos, Solitons and Fractals, vol. 21, no. 4, pp. 977-981, 2004.

[11] A. A. Himonas, G. Misiołek, G. Ponce, and Y. Zhou, "Persistence properties and unique continuation of solutions of the Camassa-Holm equation," Communications in Mathematical Physics, vol. 271, no. 2, pp. 511-522, 2007.

[12] A. Degasperis and M. Procesi, "Asymptotic integrability," in Symmetry and Perturbation Theory, A. Degasperis and G. Gaeta, Eds., pp. 23-37, World Scientific, Singapore, 1999.

[13] D. D. Holm and M. F. Staley, "Nonlinear balance and exchange of stability of dynamics of solitons, peakons, ramps/cliffs and leftons in a $1+1$ nonlinear evolutionary PDE," Physics Letters. A, vol. 308, no. 5-6, pp. 437-444, 2003.

[14] Y. Zhou, "Blow-up phenomenon for the integrable DegasperisProcesi equation," Physics Letters A, vol. 328, no. 2-3, pp. 157-162, 2004.

[15] Y. Zhou, "On solutions to the Holm-Staley $b$-family of equations," Nonlinearity, vol. 23, no. 2, pp. 369-381, 2010.

[16] Z. Guo, "Blow up, global existence, and infinite propagation speed for the weakly dissipative Camassa-Holm equation," Journal of Mathematical Physics, vol. 49, no. 3, Article ID 033516, 2008.

[17] Z. Guo, "Some properties of solutions to the weakly dissipative Degasperis-Procesi equation," Journal of Differential Equations, vol. 246, no. 11, pp. 4332-4344, 2009.

[18] W. Niu and S. Zhang, "Blow-up phenomena and global existence for the nonuniform weakly dissipative $b$-equation," Journal of Mathematical Analysis and Applications, vol. 374, no. 1, pp. 166-177, 2011.

[19] M. Zhu and Z. Jiang, "Some properties of solutions to the weakly dissipative $b$-family equation," Nonlinear Analysis: Real World Applications, vol. 13, no. 1, pp. 158-167, 2012. 


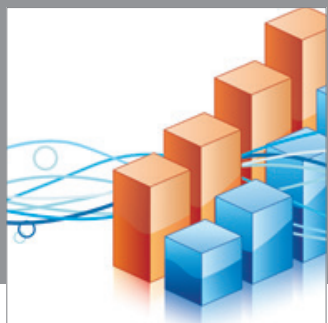

Advances in

Operations Research

mansans

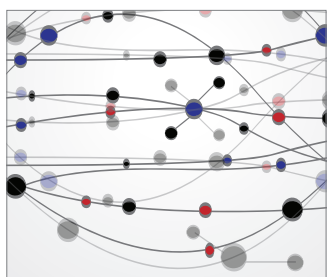

The Scientific World Journal
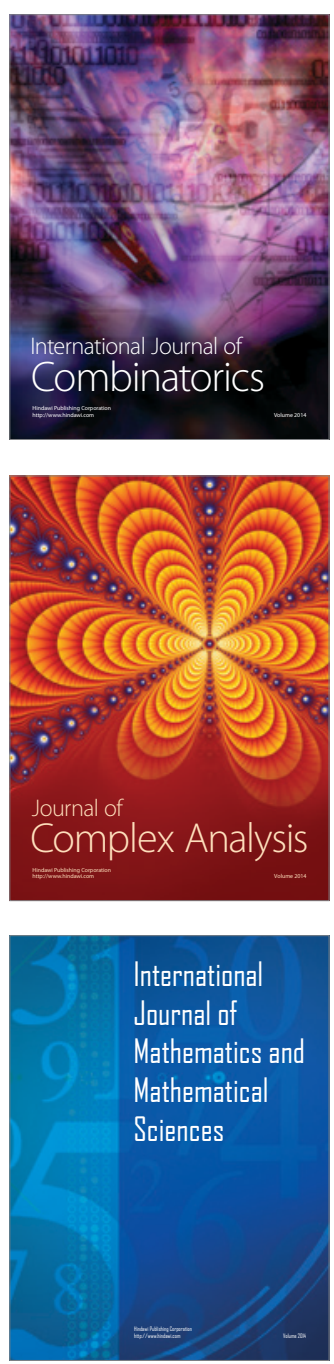
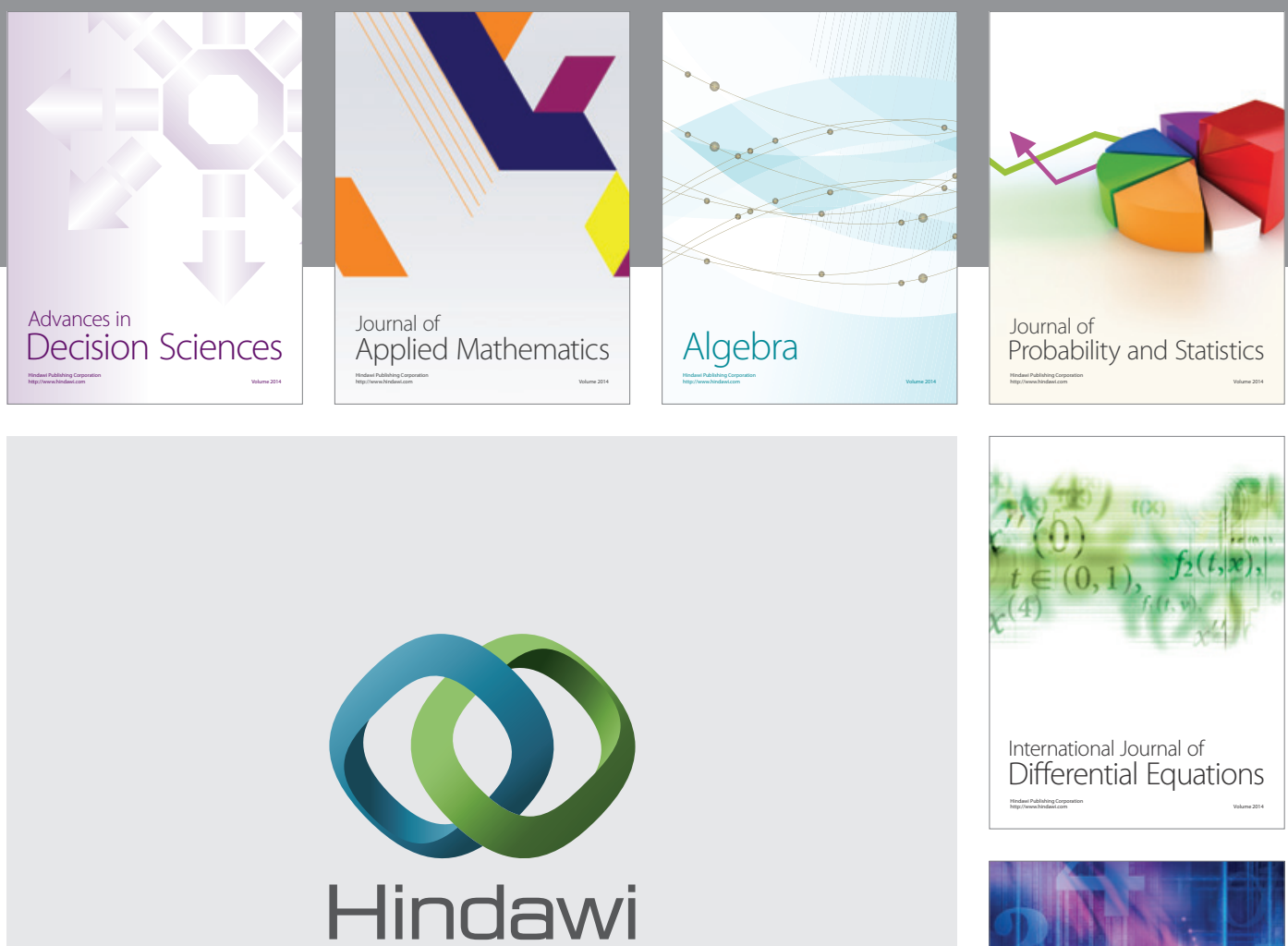

Submit your manuscripts at http://www.hindawi.com
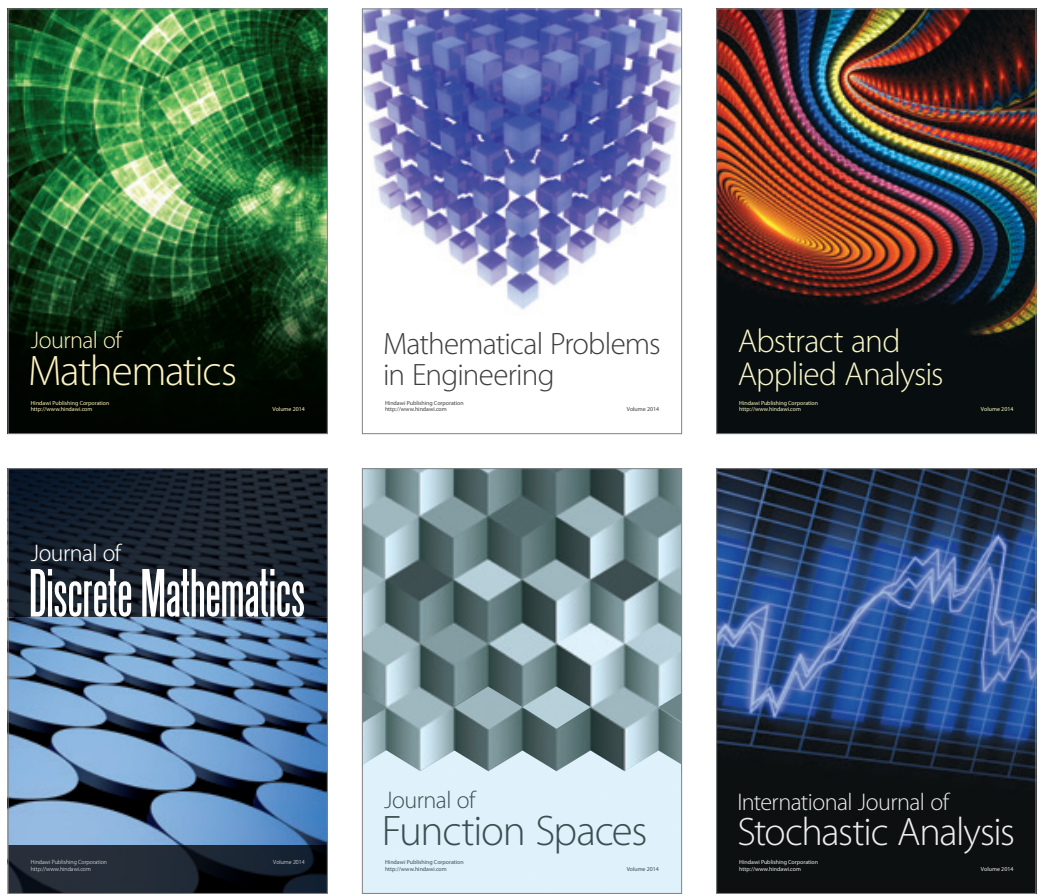

Journal of

Function Spaces

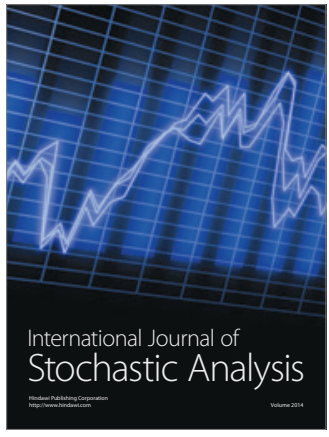

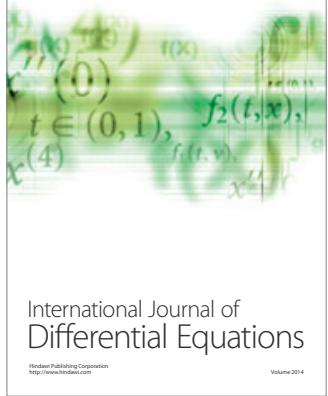
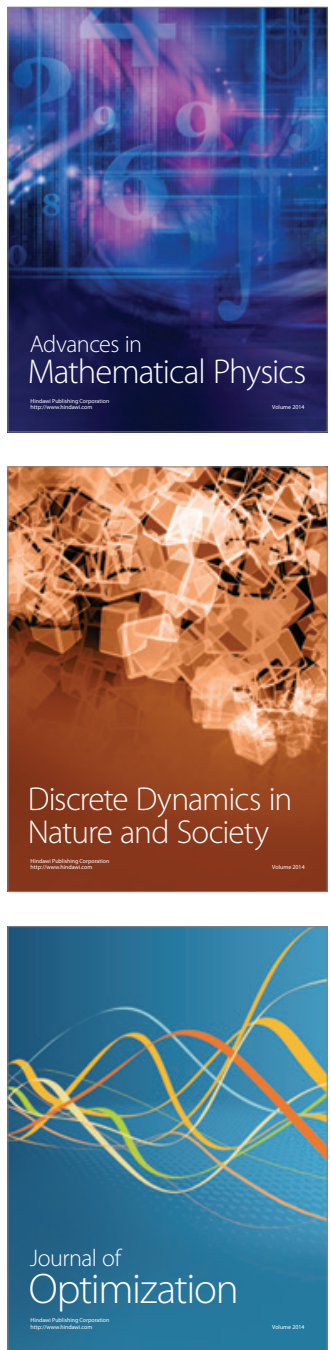